\title{
Experimental and procedural pain responses in primary dysmenorrhea: a systematic review
}

\author{
This article was published in the following Dove Press journal: \\ Journal of Pain Research \\ 12 September 2017 \\ Number of times this article has been viewed
}

\section{Laura A Payne' \\ Andrea J Rapkin ${ }^{2}$ \\ Laura C Seidman' \\ Lonnie K Zeltzer' \\ Jennie CITsao'}

'Pediatric Pain and Palliative Care Program, ${ }^{2}$ Department of Obstetrics and Gynecology, David Geffen School of Medicine at UCLA, Los Angeles, CA, USA
Correspondence: Laura A Payne Pediatric Pain and Palliative Care Program, David Geffen School of Medicine at UCLA, 10833 Le Conte Avenue, 22-464 MDCC, Los Angeles, CA 90095-1752, USA

$\mathrm{Tel}+\mathrm{I} 3108256953$

Email LPayne@mednet.ucla.edu

\begin{abstract}
Primary dysmenorrhea (PD) has been the focus of a number of experimental pain studies. Although a number of reviews exist, few have critically evaluated the existing body of research on PD and experimental and procedural pain. Data from 19 published research articles that include women with PD and responses to an experimental or procedural pain stimulus (or stimuli) suggest that women with PD may have elevated pain reactivity, as compared to women without PD. This pattern appears to be true across different phases of the menstrual cycle. However, there is an abundance of conflicting findings, which may be due to significant methodological issues such as inconsistent definitions of PD, wide variation in experimental pain methodologies, and inaccurate assessment of the menstrual cycle. Future research should focus on identifying specific symptoms (i.e., pain threshold ratings) to more clearly define what constitutes $\mathrm{PD}$, establish reliable and valid laboratory testing protocols, and assess the menstrual cycle with greater precision.
\end{abstract}

Keywords: primary dysmenorrhea, menstrual pain, acute pain, menstrual cycle, central pain mechanisms

\section{Introduction}

Dysmenorrhea, or menstrual pain, is a recurrent and disabling condition. Primary dysmenorrhea (PD) refers to menstrual-related pain without identified organic pathology, whereas "secondary" dysmenorrhea is menstrual pain due to an identified medical condition such as endometriosis, uterine fibroids, or pelvic inflammatory disease.

Severe PD leading to medication use and absenteeism affects up to $25 \%$ of the female population $^{1}$ and can have a significant impact on quality of life. ${ }^{2-4}$ Recent research has suggested that PD may contribute to the development of subsequent chronic pain disorders; ${ }^{5,6}$ therefore a more complete understanding of this condition will facilitate improved management of PD and ultimately the prevention of future chronic pain syndromes. However, despite its high prevalence and notable impact, little is known about how and why PD occurs in some girls and women. ${ }^{7}$

For decades, research on human pain has relied on the systematic administration of acute pain stimuli in a laboratory or clinical setting. This approach is advantageous, primarily because stimuli used to induce pain can be controlled by the experimenter and responses may be evaluated through self-report, and by psychophysiological or biophysical measures. Experimental pain research also allows for standardization of procedures across time points, settings, and populations. ${ }^{8}$ Various standardized pain induction procedures have been utilized among many different chronic pain syndromes, such as fibromyalgia ${ }^{9-11}$ or headache. ${ }^{12-14}$ However, a major drawback of 
these procedures is whether the findings can be generalized to naturally occurring pain, where context and other factors are influential. ${ }^{15}$

When assessing pain reactivity, it is common to determine pain sensitivity and tolerance in areas of referred pain - in PD, this constitutes primarily the abdomen, low back, or thighs, as it may be traumatic or risky to instrument the cervix and uterus in girls and young women. Localized hyperalgesia in these areas has been demonstrated in a number of pelvic pain conditions such as chronic pelvic pain (CPP), endometriosis, and bladder pain. ${ }^{16-19}$ However, assessment of pain reactivity in other anatomic locations outside the areas of referred pain can reveal alterations in pain processing in the spinal cord and brain, potentially providing evidence of central sensitization. "Central sensitization" manifests as hypersensitivity to pain occurring outside of the referred area of pain ${ }^{20-22}$ and has been implicated in various pelvic pain conditions, including CPP, ${ }^{23}$ bladder pain, ${ }^{24}$ irritable bowel syndrome, ${ }^{25}$ and endometriosis. ${ }^{23,26}$ Central sensitization is usually accompanied by allodynia and hyperalgesia, and may be localized to one specific region or be widespread. Determining whether or not women with PD show markers of central sensitization may help guide the development of novel treatments, reduce suffering, and, ideally, help prevent future chronic pain problems.

Research over the past several decades suggests heightened pain reactivity and deficits in central pain processing in PD. Although a number of reviews address current knowledge about the etiology or management of $\mathrm{PD},{ }^{27-31}$ no review has focused solely on experimental pain studies in this population. The purpose of this article is to review existing experimental pain findings from studies that include at least a subgroup of participants with PD.

\section{Methods}

We conducted literature searches using the PubMed and Web of Science electronic databases from the beginning of each database through May 24, 2017. Based on the procedures of a recent review, ${ }^{27}$ search terms combined "dysmenorrhea" with each of the following additional terms: experimental pain, laboratory pain, hyperalgesia, pain threshold, pain tolerance, pain sensitivity, pain reactivity, pain perception, central sensitization, and conditioned pain modulation. These searches yielded a total of 806 records, of which 444 remained after duplicates were removed. Three records were immediately excluded due to a lack of an English language abstract, and 392 were excluded after abstract review for the following reasons: study based on an animal model, study did not include an experimental pain stimulus, or study did not include a population with PD. The full text of the remaining 49 studies was examined, and an additional 28 studies were excluded for the reasons identified earlier, leaving 21 studies that met inclusion criteria. Cross-checking the reference lists of identified articles yielded one additional study that met inclusion criteria and was added. Of note, two studies did not include a comparison group, ${ }^{32,33}$ and one additional study did not report group comparisons, ${ }^{34}$ so these three studies are not described further in the review. The PRISMA diagram depicting the study selection process is shown in Figure 1. Therefore, a total of 19 studies were included in this systematic review (Table 1). An overview of study methodological characteristics is presented in Table 2 . Results presented in the following section have been categorized by findings of studies that stimulated 1) areas of referred pain and 2) areas remote to referred pain. In addition, within each of these categories, results are described separately for when participants were tested during menstrual and non-menstrual phases.

\section{Results}

\section{Studies stimulating areas of referred pain}

The experience of menses triggers referred pain in women with PD (including the low back, abdomen, and possibly the thighs), and given the risks of direct uterine stimulation, it is reasonable to examine pain reactivity to an experimental stimulus applied to these areas of referred pain or the cervix, that are accessible and share some innervations with the uterine body. The studies described in the following paragraphs focus on results from experimental acute pain testing in areas of referred pain during menstruation and across the menstrual cycle.

\section{Menstrual phase testing}

Extant data using common, standardized pain induction methods such as heat, cold, pinch, and pressure pain have examined the hypothesis that women with PD experience greater pain sensitivity and lower pain tolerance and pain threshold in areas of referred menstrual pain during menstruation (Table 1). Lower pain thresholds for electrical stimuli to the muscle and subcutis layers occurred around the time of menstruation (for women with "and" without PD), but only women with PD also demonstrated this effect on the skin. ${ }^{35}$ Women with PD also evidenced greater hyperalgesia in the abdominal muscle layer compared to the subcutaneous tissue or skin, and the degree of abdominal hyperalgesia during menstruation was significantly correlated with self-reported levels of menstrual pain. ${ }^{35}$ Heat, 


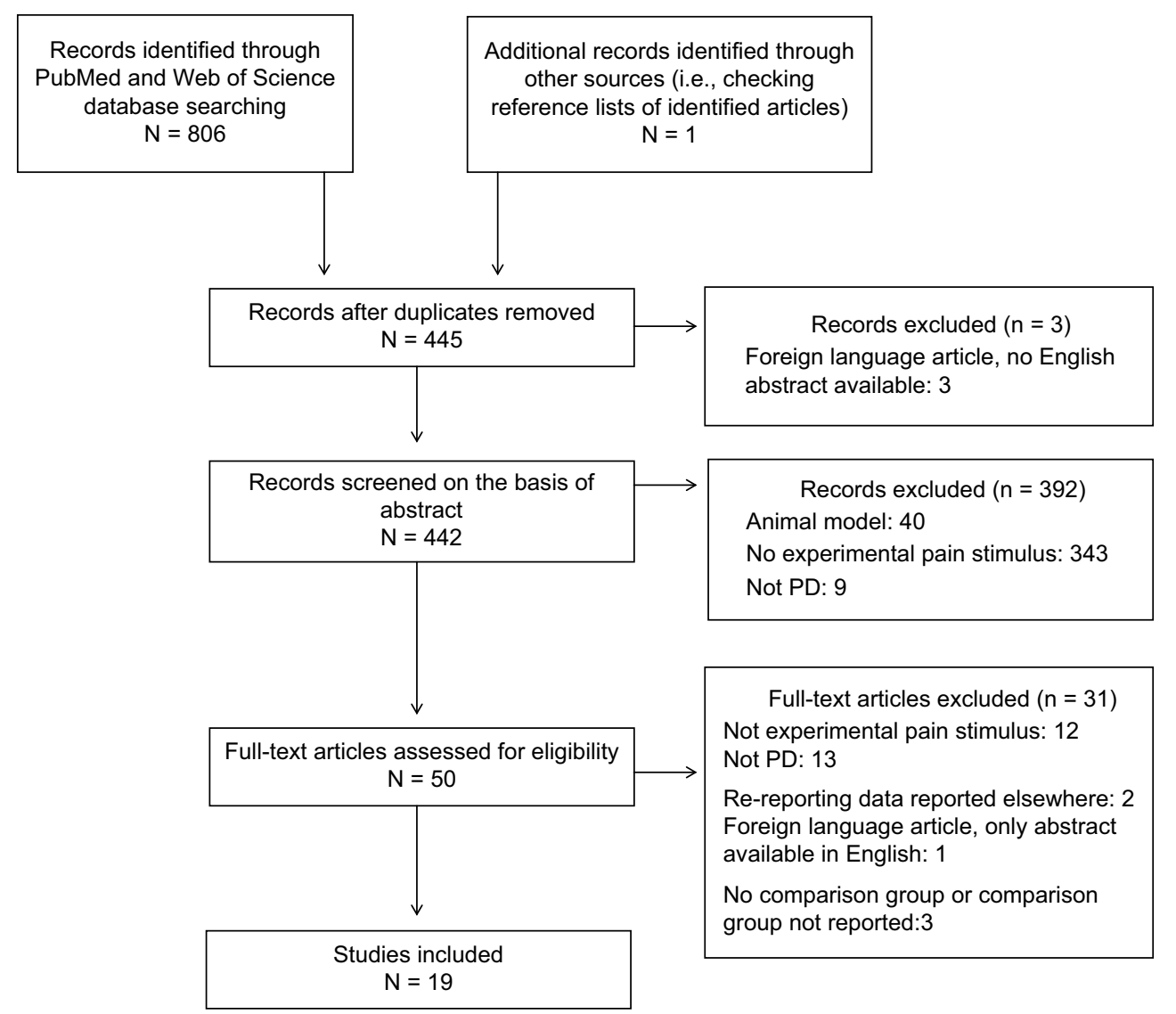

Figure I PRISMA flowchart of study selection process.

Abbreviations: PD, primary dysmenorrhea; PRISMA, Preferred Reporting Items for Systematic Reviews and Meta-Analyses..

Table I Summary of findings from laboratory pain studies involving PD

\begin{tabular}{|c|c|c|c|}
\hline Study & Menstrual cycle phase & Results $^{ \pm}$(type of pain stimulus and outcome) & $\begin{array}{l}\text { Location of pain } \\
\text { stimulation }\end{array}$ \\
\hline \multirow[t]{2}{*}{ Wei et $\mathrm{al}^{39}$} & Menstrual & Heat: $\mathrm{PD} \geq \mathrm{HC}$ & Abdomen, forearm \\
\hline & Periovulatory & Heat: PD $\geq \mathrm{HC}$ & \\
\hline Payne et $\mathrm{a}^{33}$ & Did not measure & Cold: no comparison group & Right hand \\
\hline \multirow{2}{*}{ Slater et al $\left.\right|^{43, *}$} & Did not measure & Cold: severe and moderate $D \geq$ no pain or mild $D$ & Dorsal wrist, lumbar spine, leg, \\
\hline & & Pressure: severe and moderate $D \geq$ no pain or mild $D$ & upper trapezius, wrist \\
\hline \multirow[t]{4}{*}{ Lee et $\mathrm{al}^{38}$} & Menstrual & Heat: $P D=H C$ & Abdomen, forearm \\
\hline & & Cold: $\mathrm{PD}=\mathrm{HC}$ & \\
\hline & Periovulatory & Heat: $P D=H C$ & \\
\hline & & Cold: $\mathrm{PD}=\mathrm{HC}$ & \\
\hline \multirow[t]{2}{*}{ lacovides et $\mathrm{a}^{47, * *}$} & Menstrual & Ischemic: PD > HC & Forearm \\
\hline & Late follicular & Ischemic: PD > HC & \\
\hline Arendt-Nielsen et al $4^{40}$ & Mid-follicular & Distension: PD > HC & Uterine cervix \\
\hline Kaislasuo et $\mathrm{al}^{42, *}$ & Postmenstrual & Insertion: severe $\mathrm{D}>$ disturbing or none/mild $\mathrm{D}$ & Uterine cavity \\
\hline Molins-Cubero et al ${ }^{32}$ & Menstrual & Pressure: no comparison group & Sacroiliac joints \\
\hline \multirow[t]{3}{*}{ Tu et $\mathrm{al}^{48, *}$} & Menstrual & Distension: highest quartile $D>$ lower three quartiles & Bladder \\
\hline & Follicular & Distension: highest quartile $\mathrm{D}>$ lower three quartiles & \\
\hline & Luteal & Distension: highest quartile $\mathrm{D}>$ lower three quartiles & \\
\hline \multirow[t]{3}{*}{ lacovides et $\mathrm{al}^{37}$} & Menstrual & Injection: $\mathrm{PD}>\mathrm{HC}$ & Lower back, forearm \\
\hline & Follicular & Injection: $\mathrm{PD}>\mathrm{HC}$ & \\
\hline & Luteal & Injection: $\mathrm{PD}>\mathrm{HC}$ & \\
\hline Ye et $\mathrm{al}^{6}$ & $\begin{array}{l}\text { Within I week following end of } \\
\text { menstruation }\end{array}$ & $\begin{array}{l}\text { Orthodontic: severe } \mathrm{PD}>\text { moderate } \mathrm{PD}>\text { mild } \mathrm{PD} \\
\mathrm{CPT} \text { : severe } \mathrm{PD}=\text { moderate } \mathrm{PD}=\text { mild } \mathrm{PD}\end{array}$ & Mouth, hand \\
\hline
\end{tabular}


Table I (Continued)

\begin{tabular}{|c|c|c|c|}
\hline Study & Menstrual cycle phase & Results $^{ \pm}$(type of pain stimulus and outcome) & $\begin{array}{l}\text { Location of pain } \\
\text { stimulation }\end{array}$ \\
\hline \multirow[t]{6}{*}{ Vincent et al ${ }^{4}$} & Menstrual & Heat: PD > HC & Lower abdomen, forearm \\
\hline & & Brain activation: $\mathrm{PD}=\mathrm{HC}$ & \\
\hline & & Brain deactivation: $\mathrm{PD}>\mathrm{HC}$ & \\
\hline & Ovulatory/luteal (data & Heat: PD > HC & \\
\hline & combined) & Brain activation: $\mathrm{PD}>\mathrm{HC}$ & \\
\hline & & Brain deactivation: $\mathrm{PD}=\mathrm{HC}$ & \\
\hline \multirow[t]{2}{*}{ Brinkert et $\mathrm{al}^{41}$} & "Mid-cycle" & Electrical: $\mathrm{PD}=\mathrm{HC}$ & Back of neck, mid-back, low \\
\hline & & Distension: $\mathrm{PD}>\mathrm{HC}$ & back, sigmoid colon, rectum \\
\hline \multirow[t]{4}{*}{ Bajaj et $\mathrm{a}^{36}$} & Menstrual ${ }^{\$}$ & Heat: $P D \geq H C$ & Abdomen, low back, arm, thigh \\
\hline & & Pressure: $\mathrm{PD} \geq \mathrm{HC}$ & \\
\hline & & Pinch: $P D=H C$ & \\
\hline & & Tactile: $\mathrm{PD}=\mathrm{HC}$ & \\
\hline \multirow[t]{8}{*}{ Granot et $\mathrm{al}^{45}$} & Menstrual & Heat: $P D=H C$ & Hand \\
\hline & & Laser: $\mathrm{PD} \geq \mathrm{HC}$ & \\
\hline & Mid-follicular & Heat: $P D=H C$ & \\
\hline & & Laser: $\mathrm{PD} \geq \mathrm{HC}$ & \\
\hline & Ovulatory & Heat: $P D=H C$ & \\
\hline & & Laser: $\mathrm{PD} \geq \mathrm{HC}$ & \\
\hline & Mid-luteal & Heat: $\mathrm{PD}=\mathrm{HC}$ & \\
\hline & & Laser: $\mathrm{PD} \geq \mathrm{HC}$ & \\
\hline \multirow[t]{4}{*}{ Giamberardino et $\mathrm{a}^{35}$} & Menstrual & Electrical: $\mathrm{PD} \geq \mathrm{HC}$ & Abdomen, leg, arm \\
\hline & Periovulatory & Electrical: $\mathrm{PD} \geq \mathrm{HC}$ & \\
\hline & Luteal & Electrical: $\mathrm{PD} \geq \mathrm{HC}$ & \\
\hline & Premenstrual & Electrical: $\mathrm{PD} \geq \mathrm{HC}$ & \\
\hline Amodei and Nelson- & Menstrual & Pressure: $\mathrm{PD}=\mathrm{HC}$ & Finger, arm \\
\hline \multirow[t]{5}{*}{ Gray $^{51}$} & & Ischemic: PD = HC & \\
\hline & Intermenstrual & Pressure: $\mathrm{PD}=\mathrm{HC}$ & \\
\hline & & Ischemic: PD = HC & \\
\hline & Premenstrual & Pressure: $\mathrm{PD}=\mathrm{HC}$ & \\
\hline & & Ischemic: PD = HC & \\
\hline Hapidou and De & Follicular & CPT: $\mathrm{PD}<\mathrm{HC}$ & Arm \\
\hline Catanzaro ${ }^{50}$ & Luteal & CPT: PD = HC & \\
\hline \multirow[t]{4}{*}{ Goolkasian $^{46}$} & Menstrual & Heat: PD $=\mathrm{HC}$ & Forearm \\
\hline & Postmenstrual & Heat: $P D=H C$ & \\
\hline & Ovulatory & Heat: $P D=H C$ & \\
\hline & Premenstrual & Heat: $P D=H C$ & \\
\hline \multirow[t]{2}{*}{ Aberger et $\mathrm{al}^{49}$} & Menstrual/Premenstrual/ & Ischemic: PD = HC & Arm \\
\hline & Postmenstrual ${ }^{\&}$ & & \\
\hline Cox and Meyer ${ }^{34}$ & Not stated & Ischemic: not reported & Upper arm \\
\hline Haman $^{44, *}$ & Not stated & Pressure: $\mathrm{D}>\mathrm{HC}$ & Finger \\
\hline
\end{tabular}

Notes: ${ }^{ \pm}$Symbols $(\geq,>,=,<)$indicate direction of effect, e.g., PD $\geq \mathrm{HC}$ indicates that the PD group had "equivalent or greater" pain responses than the HC group. $*$ These samples included participants with primary and secondary dysmenorrhea. **These were the same participants as in lacovides et al. ${ }^{37} \$$ Testing on PD only was also conducted

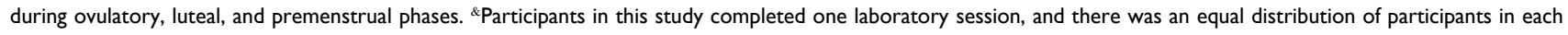
phase of the menstrual cycle.

Abbreviations: CPT, cold pressor test; D, dysmenorrhea (including primary and possibly secondary dysmenorrhea); HC, healthy control; PD, primary dysmenorrhea.

pain, and pressure pain thresholds on the abdomen and low back have also been shown to be significantly lower in women with PD during the menstrual phase as compared to menstruating women without PD. ${ }^{4,36}$ Analogously, following a saline injection into muscle of the low back, women with PD reported higher peak pain and longer duration of pain responses to the injection during menstruation compared to those women without PD. ${ }^{37}$
Only two published studies have not shown differences between women with and without PD in response to pain stimulation in areas of referred pain during menstruation. The first, which compared 99 Asian women with PD to 101 Asian healthy controls, found no group differences in heat pain thresholds on the abdomen during menstruation. ${ }^{38}$ Similarly, one recent study found lower heat pain thresholds in women with PD compared to women without PD when 


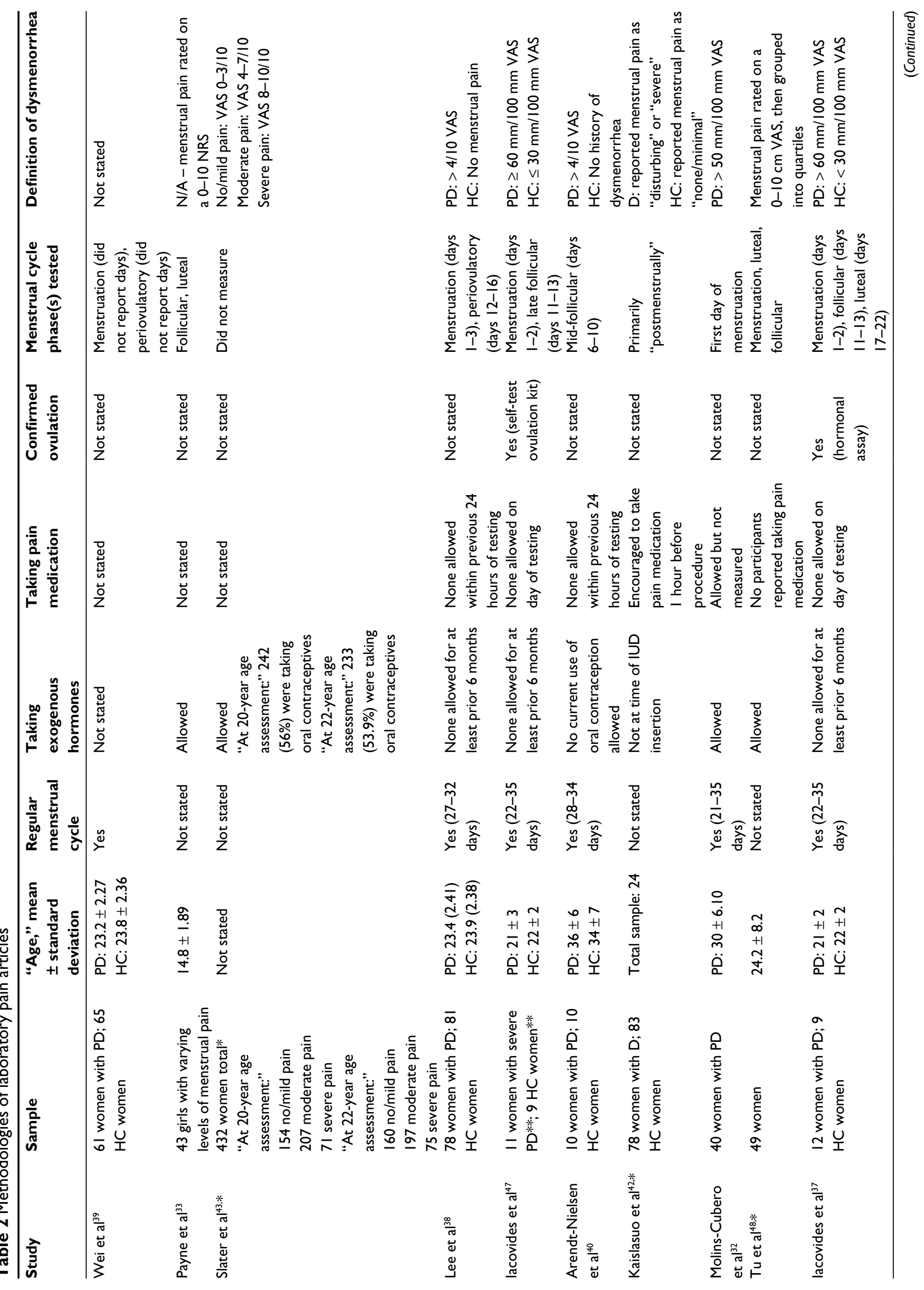




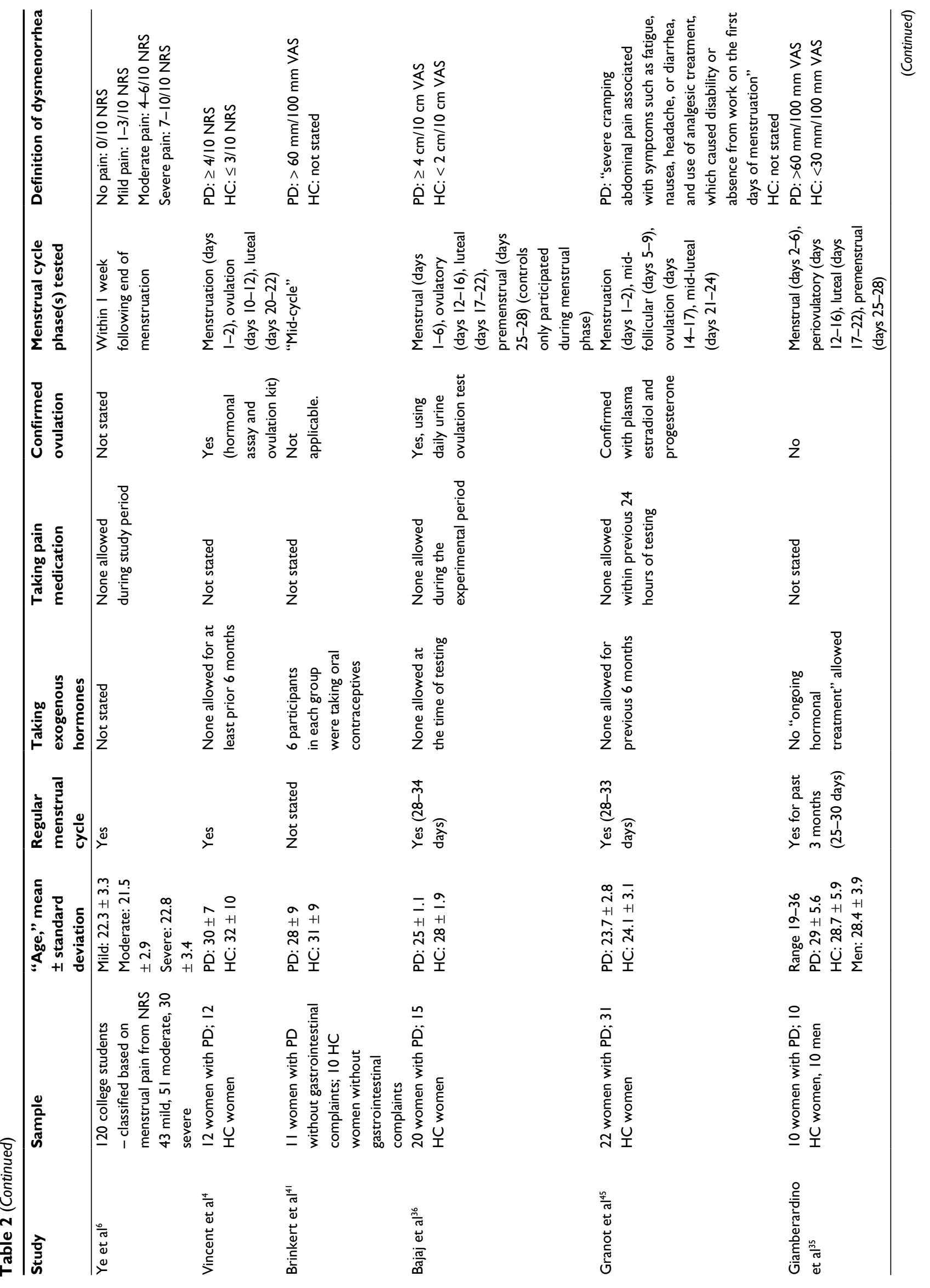




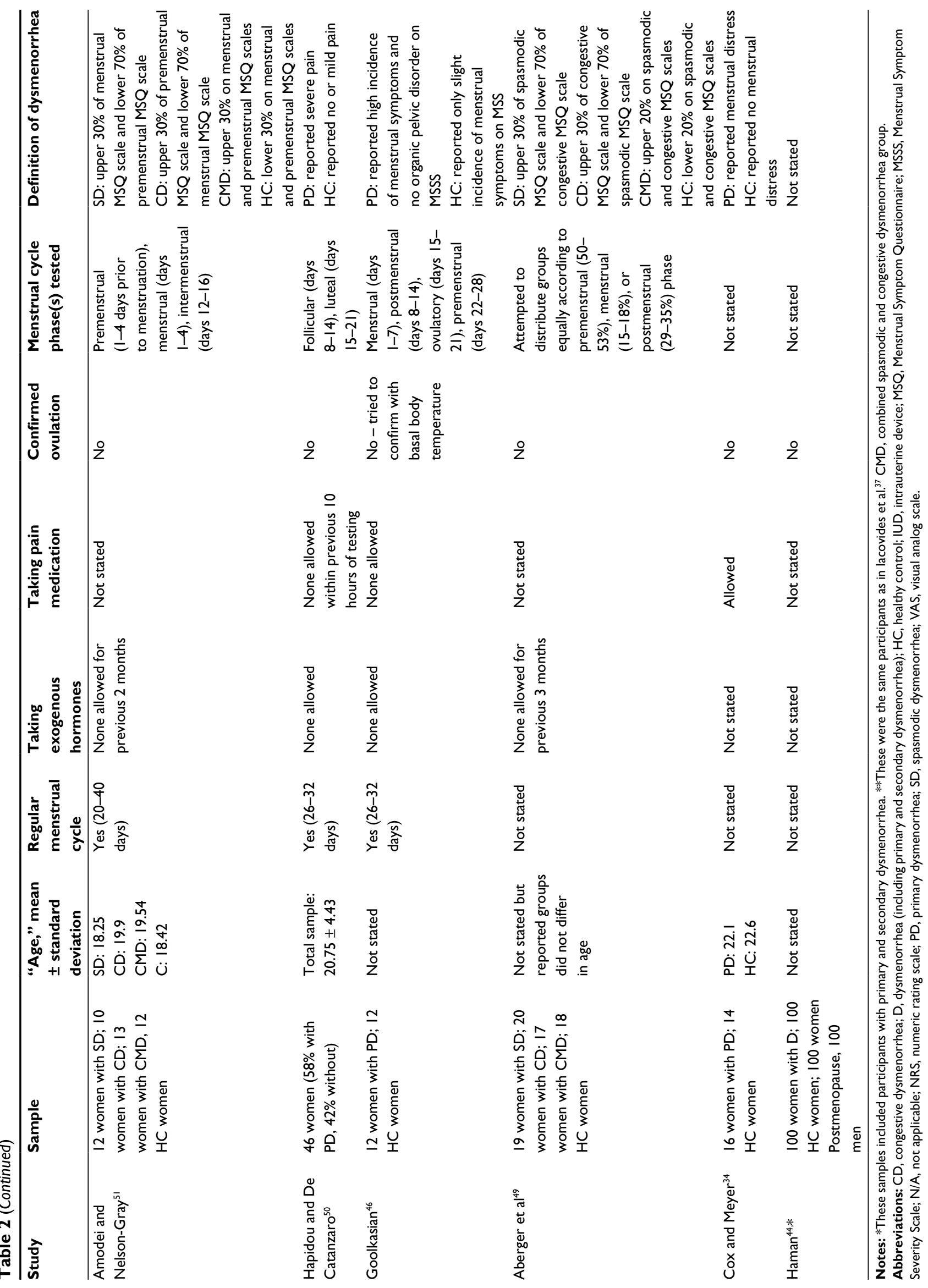


tested on the abdomen, although these differences did not reach statistical significance. ${ }^{39}$

\section{Non-menstrual phase testing}

In a pain testing paradigm that involved distension of the uterine cervix during the mid-follicular phase, women with PD showed increased overall pain ratings and larger body areas experiencing pain from the distension, ${ }^{40}$ compared to women without PD. Pain intensity ratings were significantly higher, and bag volumes required to reach a pain rating of 7 out of 10 were significantly lower in the PD group. Furthermore, prolonged bag distension was associated with an increase in pain ratings for the PD group, whereas a decrease in pain ratings was observed in the control group. Lower pain thresholds for sigmoid colon and rectal distension have also been reported in women with PD (when tested only in "midcycle"), ${ }^{41}$ and women with PD also have shown elevated pain reactivity to a saline injection in the low back during follicular and luteal phases. ${ }^{37}$ Women with severe dysmenorrhea also report higher pain during the postmenstrual insertion of an intrauterine device, as compared to women who categorized their dysmenorrhea symptoms as "disturbing" or "none/ mild." 42 Similarly, Vincent et $\mathrm{al}^{4}$ found that women with menstrual pain had significantly lower pain thresholds on the abdomen during ovulatory/luteal phases (data combined). A recent study with a large sample of women found a significant relationship between menstrual pain severity and pressure pain thresholds on the lumbar spine in 432 women with varying levels of PD (menstrual cycle phase was not assessed). ${ }^{43}$

As with the results described in menstrual phase testing earlier, only a small group of studies has failed to find significant group differences in non-menstrual phases of the menstrual cycle. One study found that pain threshold and tolerance to electrical stimulation in the low back were "highest" during mid-cycle (typically the periovulatory phase - days 12-16; testing was not conducted during menstruation in this study), and this pattern was true for both women with "and" without PD. ${ }^{41}$ Women with and without PD have also shown similar heat and cold pain thresholds when tested on the abdomen during the periovulatory period. ${ }^{38,39}$

\section{Conclusion}

Overall, results suggest enhanced pain sensitivity in areas of referred pain (as measured by decreased threshold and tolerance) in women with PD compared to women without PD, "across the menstrual cycle." However, this was not entirely consistent. Brinkert et $\mathrm{al}^{41}{ }^{41}$ for example, found significant group differences when testing distension of the sigmoid colon and rectum but not when testing electrical stimulation to the skin. The authors suggest that dysmenorrhea may be associated with somatic hyperalgesia in deep tissue only, as central sensitization is not evident in the skin. This idea has been supported by two other studies in women with PD. ${ }^{35,38}$ However, a number of other studies described earlier have found significant group differences when pain stimuli are applied to the skin. Variations in type and intensity of stimuli used may account for some of these seemingly contradictory findings. For example, Lee et $\mathrm{al}^{38}$ and Wei et $\mathrm{al}^{39}$ restricted the temperature range on the heat thermode from $0^{\circ} \mathrm{C}$ to $50^{\circ} \mathrm{C}$, which may have unintentionally created a ceiling effect due to a low maximum temperature. However, generally, results suggest that women with PD do experience hypersensitivity to areas of referred pain.

\section{Studies stimulating body locations separate from areas of referred pain}

The earliest study of laboratory pain reactivity in women with PD sought to answer the question as to "whether dysmenorrheic patients actually receive more intense pain stimuli than do non-dysmenorrheics, or whether they are merely more sensitive to pain than their more fortunate sisters." ${ }^{44}$ Determining the nature of pain responsivity in areas of the body unrelated to uterine/abdominal pain may help clarify whether differences in overall pain processing exist in women with $\mathrm{PD}$.

\section{Menstrual phase testing}

A number of research investigations involving electrical stimulation, heat pain, laser pain, pressure pain, and pinch pain have demonstrated significant differences in pain threshold between women with and without PD in body regions outside the abdomen/low back during menstruation. . $3,36,45,46$ In the very first published study of pain responses in PD, differences in pain threshold in response to the pressure of a weight placed on the thumb were compared in women with and without PD (as well as a group of postmenopausal women and a group of men). The author reported that during menses, women with PD had lower pain thresholds than any of the other groups. ${ }^{44}$ Women with PD have also reported higher peak pain during the menstrual phase than women without PD following a saline injection into the forearm, ${ }^{37}$ and in a subsequent study testing muscle ischemia, the authors found that women with PD reported higher pain ratings compared to controls during menstruation. ${ }^{47}$ Women with $\mathrm{PD}$ have higher heat pain thresholds when tested on the forearm during menstruation, ${ }^{39}$ and report more pain from a bladder distension during menstruation, ${ }^{48}$ when compared to women without PD. 
We were able to identify only one study that did not find significant differences between women with and without PD in areas outside of referred pain during menstruation. That study included 56 women with menstrual pain and 18 women without menstrual pain and subjected them to ischemic pain by using a blood pressure cuff on the nondominant arm. No significant differences in somatic pain threshold or tolerance were found. ${ }^{49}$

\section{Non-menstrual phase testing}

One study reported significantly lower thresholds to cold pain on the arm in women with PD in the luteal phase only. ${ }^{50}$ Significant negative associations between menstrual pain ratings and cold pain thresholds tested on the wrist and hand have also been reported (cycle phase was not assessed), although no associations were found between menstrual pain and leg, upper trapezius, and wrist pressure pain. ${ }^{43}$ Goolka$\operatorname{sian}^{46}$ tested 12 women with PD and 12 women without PD across the menstrual cycle using a radiant heat stimulus to the right forearm. For high-intensity stimuli, women with PD consistently rated the stimuli more painful than controls across all phases of the menstrual cycle, while women without PD were able to better discriminate painful stimuli during the ovulation phase. The author suggests that the findings provide conclusive evidence of group sensory differences in pain responses across the menstrual cycle. ${ }^{46}$

Granot et $\mathrm{al}^{45}$ assessed women with and without PD during mid-follicular, ovulation, and mid-luteal phases in their responses to heat pain applied to the nondominant hand from quantitative sensory testing (QST), and laser pain applied to the hand. Women in the PD group reported higher pain ratings to supra-threshold (above threshold) heat and laser pain and showed significantly longer time from the administration of the laser pain stimulus to the perception of pain. For both groups, the longest latency was recorded during the follicular phase, whereas the shortest latency was observed in the luteal phase. In a separate study, the top quartile of women with menstrual pain also reported higher pain during a bladder distension paradigm that included participants across follicular and luteal phases. ${ }^{48}$ Heat pain thresholds on the forearm are also higher in women with PD compared to women without PD in the periovulatory period. ${ }^{39}$

Finally, although not a laboratory pain paradigm, a study of an acute pain stimulus in a clinical setting attempted to provide a "naturalistic" perspective regarding how PD influences pain reactivity. ${ }^{6} \mathrm{~A}$ total of 120 college students who were scheduled to undergo a dental procedure involving an archwire placement were initially classified as having either mild, moderate, or severe PD based on their self-report responses. The severity of menstrual pain significantly correlated with pain ratings and duration of pain following the archwire placement, again supporting the notion that PD may be an indicator or result of overall pain sensitization.

Several studies that included other cycle phase testings have not found differences in thermal pain, focal pressure, and ischemic pain stimuli. ${ }^{38,46,51}$ Aberger et al ${ }^{49}$ compared women with PD with unaffected women in an ischemic pain test (inflated blood pressure cuff) measuring pain threshold and tolerance and reported no statistically significant differences between the groups of participants. The only effect of menstrual cycle phase was increased pain threshold during the premenstrual phase for the entire sample of women (collapsing the PD and non-PD groups together). Subsequent studies using ischemic pain and electrical stimulation also found no differences in pain threshold or tolerance in any menstrual cycle phase. ${ }^{35,51}$ Interestingly, women with PD have also been found to rate cold pressor pain intensity significantly "lower" than ratings of women without PD during the follicular phase only. ${ }^{50}$ The authors attribute this counterintuitive finding to the possibility that women with PD are comparing cold pressor pain to menstrual pain and therefore rating it as less severe.

\section{Conclusion}

The data present in this article suggest increased pain reactivity in areas outside of referred pain in women with PD, as compared to women without, and this pattern appears to extend beyond the menstrual phase. During menstruation, only one study failed to find group differences in response to ischemic pain, ${ }^{49}$ and this provides strong support for the notion of the association of dysmenorrhea with central sensitization, where heightened pain reactivity is experienced in areas of the body not related to the location of referred pain. However, in non-menstrual cycle phases, the findings are less clear. Significant variations in anatomic locations for pain testing may contribute to inconsistent findings. Contradictory findings using similar pain stimuli (heat and cold pain) are evident and suggest that methodological issues do not solely account for the differences. However, it is notable that studies examining ischemic pain and electrical pain did not find group differences during non-menstrual cycle phases. It is possible that these methods of pain stimulation are not sufficient to access the central pain pathways that lead to central sensitization. In addition, compared to the relatively consistent findings in the menstrual phase described earlier, it is possible that the "background" pain that participants with 
PD experience when tested during menstruation is sufficient to activate central pain networks and result in widespread pain sensitivity.

\section{Discussion}

The first part of this systematic review focused on pain reactivity in regions of referred uterine pain, including the abdomen, low back, and uterine cavity and cervix during both menses and non-menstrual phases of the cycle. Results varied across type and location of pain stimulus, but generally during menses, women with PD demonstrated increased pain reactivity compared to unaffected women in both the uterine cervix and areas of referred pain. Women with PD also tended to show increased pain reactivity across the menstrual cycle, However, several studies failed to find increased reactivity in areas of referred pain in women with PD, either during menses or during other cycle phases. . $^{4,36-38,41}$

The second half of the review examined pain reactivity in other regions, outside of the area of referred pain both during menses and across the menstrual cycle. These body areas included the arms, wrists, legs (although assessment of pain responses on the legs may be considered an area of referred pain due to overlapping dermatomes, this would vary across individuals), upper back, and mouth across a variety of pain stimuli. The evidence suggests that women with PD may show heightened pain reactivity in other body regions across the menstrual cycle, although there was more variability in these outcomes. Differences in findings could be attributable to the wide range of pain stimuli, variation in anatomic locations in areas of non-referred pain, and absence of "background" pain when tested outside of menstruation. These study differences make it difficult to draw significant linear conclusions.

\section{Methodological issues and advancing an ideal methodology}

The studies described in this review use a wide range of methodological approaches to pain assessment. In addition, a number of studies failed to include important information about the study participants, including how they were identified. These issues may be a significant source of inconsistent findings. We highlight these specific methodological concerns that may affect research on pain reactivity in women with PD by addressing 1) inconsistent definitions of PD; 2) variations in methodological procedures, such as differences in pain stimuli, methods of assessing pain responses, and lack of demographic information to characterize participants; 3 ) nonscientific or lack of assessment of cycle phase; and 4) inconsistent reporting of medication use. Each of these is reviewed in detail (Table 3), and possible solutions to each specific issue are suggested.

\section{Inconsistent classification of PD}

A critical problem is the lack of consensus regarding the threshold of pain severity allowing classification as PD. Most studies have not reported the level of pain using either a visual analog scale (VAS) or numeric rating scale (NRS) required to be classified as having PD. ${ }^{44,45}$ For studies where menstrual pain was rated using a VAS or NRS, no consistent scales (e.g., $0-10$ versus $0-100$ ) nor criteria (e.g., $4 / 10$ versus 6/10) were used to categorize women with PD. ${ }^{6,35-37,40,41,47}$ Others have used responses on the Menstrual Symptom Questionnaire, ${ }^{46,49,51}$ indicating "mild/no menstrual pain" or "moderate/severe menstrual pain." ${ }^{50}$ This ambiguity suggests the possibility that some participants would be classified as having PD in some studies, while being classified as non-PD in other studies, thus making it very difficult to compare results.

Table 3 Primary methodological issues in laboratory pain studies involving PD and recommendations

\begin{tabular}{lll}
\hline Issue & Details & Recommendation \\
\hline $\begin{array}{l}\text { Inconsistent classification } \\
\text { of PD }\end{array}$ & - Menstrual pain ratings using either 0-10 or 0-100 scale & - Treat pain as a dimensional construct \\
Pain stimuli & - No consistent use of a cutoff for grouping PD & - Do not create "PD" and "no PD" groups \\
& - Wide variety of pain stimuli used makes it difficult to identify & - Identify established pain testing protocols using \\
Participant & consistent patterns & standardized machines \\
characteristics & - Lack of information on age or cycle length of participants & - Gather information consistently and report in \\
Cycle phase & - Identification of cycle phase based on "calendar method" & - Assess during painful menstruation and non- \\
& - Ovulation not clearly identified using hormonal measures or & painful phase \\
Medication use & ovulation predictor kits & - Include hormonal measures as covariates \\
& - Lack of reporting on participants using oral contraceptives or & - Exclude use of exogenous hormones in the past \\
& exogenous hormones & 3 months \\
& - Use of pain medication not specifically excluded during & - Exclude use of pain medication in 24 hours prior \\
& participation & to testing
\end{tabular}

Abbreviation: PD, primary dysmenorrhea. 
One potential way to address this issue is to treat menstrual pain as a dimensional construct rather than categorical. Using predetermined cutoff scores not based on empirical research may obscure differences in women who have moderate menstrual pain, or who do not have consistently severe menstrual pain. This subgroup may provide additional insight into the nature of pain responding in PD. In fact, evidence in this review suggests that pain responding may be related to the "degree" of menstrual pain, rather than just the presence or absence of menstrual pain. ${ }^{6,35,42,43,48}$

\section{Study methodology and exclusion of demographic information}

Studies have used a wide variety of pain stimuli, ranging from thermal pain ${ }^{4,36,45,46,50}$ to ischemic pain ${ }^{47,49,51}$ at different parts of the body. This variability in pain induction stimuli and degree of pain provoked makes it difficult to draw conclusions about the nature of pain responsivity in women with PD. Furthermore, in one study, no statistical analyses were actually conducted, so it is not entirely clear whether pain thresholds actually differed between the groups ${ }^{44}$ Other studies have not included a comparison group, so it is unclear if pain responding would be different in a non-PD population. ${ }^{32,33}$ In addition, lack of reporting basic demographic data such as age $\mathrm{e}^{43,44,46,49}$ and cycle length ${ }^{41,43,44,49}$ further limits generalizability.

Relatively reliable pain induction protocols and methods currently exist, including the cold pressor and Medoc neurosensory analyzer, which can be used to deliver consistent pain stimuli. Research in women with PD would benefit from using these methodological approaches, which would allow for 1) comparison of responses in women with PD across studies and 2) comparison of responses in women with $\mathrm{PD}$ to individuals with other cyclical or chronic pain conditions. Although more recent studies have included more information about study participants, assessing many facets of the menstrual cycle, including average cycle length, number of days of bleeding, and pain during other parts of the menstrual cycle, should be consistent information gathered in the future studies.

\section{Potentially inaccurate assessment of cycle phase}

A very concerning issue with previously published studies is the lack of accurate assessment of cycle phase. There is a large body of literature examining the extent of the influence of gonadal hormones on pain perception, ${ }^{52-56}$ and the consensus is that gonadal hormones, including the use of oral contraceptives or exogenous hormones, impacts pain perception or modulation. ${ }^{57-61}$ The failure of many studies to clearly assess menstrual cycle phase may contribute to difficulty assessing pain responses in women with and without PD. For example, the earliest study by Haman, ${ }^{44}$ Cox and Meyer, ${ }^{34}$ and Slater et $\mathrm{al}^{43}$ did not account for menstrual cycle phase at all during the testing period. However, the vast majority of other studies relied on a relatively inaccurate "calendar method," whereby day 1 of the cycle is the first day of menstruation, day 14 is the day when ovulation is assumed to occur, and day 28 is assumed to be the final day before menstruation begins (end of cycle). ${ }^{6,35,40,41,46,49-51}$ The variability in the length of the follicular phase, particularly in adolescents, is well known. Some studies have attempted to use additional indices to confirm menstrual cycle phase such as basal body temperature ${ }^{46}$ plasma sampling for estradiol and progesterone, ${ }^{4,37,45}$ or self-test ovulation kits. ${ }^{4,36,47}$ However, these tests were performed during a single-day laboratory session (as opposed to across multiple days that may better capture the precise timing of ovulation, etc.), and it is curious why no reported subjects were excluded due to lack of ovulation or hormonal measures indicating the incorrect cycle phase. Incorrect assessment of ovulation may obscure important variations in pain reactivity across the menstrual cycle, thereby preventing a more clear understanding of hormonal influences on pain in PD.

One possible option for future studies is to continue to assess participants during a painful phase (menstruation) and non-painful phase (any part of the cycle the participant identifies as non-painful), and gather hormonal measurements to include as covariates in analyses. Estradiol and progesterone can be easily gathered through salivary collection methods. The influence of gonadal hormones can then be considered, without having to rely on self-report of cycle phase, or the assumption that all participants experience 28-day cycles.

\section{Medication use}

Many studies did report that participants were not taking oral contraceptives, although this definition ranged from not taking oral contraceptives at the time of the laboratory session $^{35,36,40,41,46,50}$ to not allowing the use of oral contraceptives for the previous 2-6 months., ${ }^{47,45,47,49,51}$ Several studies did not report whether or not oral contraceptives were used by participants. ${ }^{6,44}$ If the use of hormonal contraceptives (or any exogenous hormone) is not carefully assessed, it becomes impossible to evaluate the impact of gonadal hormones on pain reactivity. In addition, only a number of studies specifically excluded the use of pain medication (e.g., nonsteroidal anti-inflammatory drugs [NSAIDs]) within 24 hours of the 
pain testing, ${ }^{6,36-38,40,45-47,50}$ or reported that no participants were taking pain medication at the time of testing. ${ }^{48}$ The lack of restriction or assessment of this variable makes it difficult to draw conclusions about the findings.

Because medication use can significantly affect results of the pain testing, it would be important to exclude participants who have used exogenous hormones within the previous 3 months. This time frame reflects the half-life of the sex steroids and allows for at least one and depending on cycle length, two natural menstrual cycles before testing, such that pain scores and cycle regularity can be assessed. Based on the half-life, NSAIDs and other analgesics should not have been used in the previous 24 hours.

\section{Limitations}

The aim of this review was to focus on laboratory and experimental pain responses in women with PD. However, it should be noted that, for a number of studies, the participants did not receive a pelvic examination or receive an ultrasound or other test that could help to determine the presence of an underlying organic pathology. The determination of PD was largely based on self-report menstrual cycle regularity and occurrence of pain only during menstruation. This leaves the possibility that some of these participants may have been experiencing dysmenorrhea secondary to an organic pathology. We also limited our search to two databases: PubMed and Web of Science. It is possible that additional studies would have been identified using other databases such as Google Scholar. In addition, four studies were excluded because the manuscripts were not published in the English language. It is possible that these studies may have provided additional data that we are not able to report on.

\section{Conclusion and future directions}

This review examined all published studies of experimental pain responses in women with $\mathrm{PD}$. Evidence from these data support the idea that women with PD demonstrate elevated pain reactivity, in areas of both referred and non-referred pain, during menstruation and at other points in the menstrual cycle. However, a substantial portion of studies did not find any group differences between women with and without PD either during menstruation or in other cycle phases. Methodological differences and variation may account for a significant part of these inconsistencies.

Understanding the factors that may be associated with underlying pain responses in women with PD may help clarify the relationship of menstrual pain to the development of future pain problems. If women with PD share common behavioral and neurobiological markers with individuals who have other chronic pain problems, this may suggest a vulnerability to develop these problems in the future. ${ }^{27}$ Laboratory assessment of acute pain responses to acute pain stimuli in a variety of pain conditions has provided many clues to the impact of social, behavioral, and biological differences in pain reactivity. These data are critical for determining the pathogenesis of PD and its relation to noncyclical pain.

Future work should focus on identifying consistent methodologies and laboratory protocols that assess the full range of pain reactivity including both excitatory and inhibitory pain processes. $^{62,63}$ In addition, it is crucial to determine appropriate inclusion criteria and markers for $\mathrm{PD}$, perhaps using a combination of a dimensional NRS and biomarkers. Objective assessment of ovulation (including ovulation predictor kits or possible ultrasound) may provide additional clues regarding the nature of the menstrual cycle and guide the timing of experimental pain assessment. Future work should also focus on inclusion of younger populations in experimental pain research, which may provide greater insight into the nature of pain responding in PD. ${ }^{27}$ Refining our research practices will provide a better understanding of the specific mechanisms contributing to menstrual pain. This knowledge will then allow for the development of targeted, appropriate interventions for girls and women with this chronic and disabling condition.

\section{Acknowledgments}

This research was supported by grants from the National Institute of Child Health and Human Development (K23HD077042; Principal Investigator: Laura A Payne), UCLA Children's Discovery and Innovation Institute (CDI Seed Grant Award; Principal Investigator: Laura A Payne), and National Center for Advancing Translational Sciences UCLA Clinical and Translational Science Institute (KL2TR000122; Principal Investigator: Laura A Payne).

\section{Disclosure}

The content is solely the responsibility of the authors and does not necessarily represent the official views of the National Institutes of Health (NIH). The authors report no conflicts of interest in this work.

\section{References}

1. Grandi G, Ferrari S, Xholli A, et al. Prevalence of menstrual pain in young women: what is dysmenorrhea? J Pain Res. 2012;5:169-174.

2. Hillen TI, Grbavac SL, Johnston PJ, Straton JA, Keogh JM. Primary dysmenorrhea in young Western Australian women: prevalence, impact, and knowledge of treatment. J Adolesc Health. 1999;25(1):40-45.

3. Nur Azurah AG, Sanci L, Moore E, Grover S. The quality of life of adolescents with menstrual problems. J Pediatr Adolesc Gynecol. 2013;26(2):102-108. 
4. Vincent K, Warnaby C, Stagg CJ, Moore J, Kennedy S, Tracey I. Dysmenorrhoea is associated with central changes in otherwise healthy women. Pain. 2011;152(9):1966-1975.

5. Hardi G, Evans S, Craigie M. A possible link between dysmenorrhoea and the development of chronic pelvic pain. Aust NZJ Obstet Gynaecol. 2014;54(6):593-596.

6. Ye R, Wang S, Li Y, et al. Primary dysmenorrhea is potentially predictive for initial orthodontic pain in female patients. Angle Orthod. 2014;84(3):424-429.

7. Berkley KJ, McAllister SL. Don't dismiss dysmenorrhea! Pain. 2011;152:1940-1941.

8. Moore DJ, Keogh E, Crombez G, Eccleston C. Methods for studying naturally occurring human pain and their analogues. Pain 2013;154(2):190-199.

9. Brusselmans G, Nogueira H, De Schamphelaere E, Devulder J, Crombez G. Skin temperature during cold pressor test in fibromyalgia: an evaluation of the autonomic nervous system? Acta Anaesthesiol Belg. 2015;66(1):19-27.

10. Huber A, Suman AL, Biasi G, Carli G. Alexithymia in fibromyalgia syndrome: associations with ongoing pain, experimental pain sensitivity and illness behavior. J Psychosom Res. 2009;66(5):425-433.

11. Stevens A, Batra A, Kotter I, Bartels M, Schwarz J. Both pain and EEG response to cold pressor stimulation occurs faster in fibromyalgia patients than in control subjects. Psychiatry Res. 2000;97(2-3):237-247.

12. Bishop KL, Holm JE, Borowiak DM, Wilson BA. Perceptions of pain in women with headache: a laboratory investigation of the influence of pain-related anxiety and fear. Headache. 2001;41(5):494-499.

13. Cathcart S, Winefield AH, Lushington K, Rolan P. Effect of mental stress on cold pain in chronic tension-type headache sufferers. J Headache Pain. 2009;10(5):367-373.

14. Ukestad LK, Wittrock DA. Pain perception and coping in female tension headache sufferers and headache-free controls. Health Psychol. 1996;15(1):65-68.

15. Rollman GB. The need for ecological validity in studies of pain and ethnicity. Pain. 2005;113(1-2):3-4.

16. As-Sanie S, Harris RE, Harte SE, Tu FF, Neshewat G, Clauw DJ. Increased pressure pain sensitivity in women with chronic pelvic pain. Obstet Gynecol. 2013;122(5):1047-1055.

17. He W, Liu X, Zhang Y, Guo SW. Generalized hyperalgesia in women with endometriosis and its resolution following a successful surgery. Reprod Sci. 2010;17(12):1099-1111.

18. Whitaker LH, Reid J, Choa A, et al. An exploratory study into objective and reported characteristics of neuropathic pain in women with chronic pelvic pain. PLoS One. 2016;11(4):e0151950.

19. Giamberardino MA, Tana C, Costantini R. Pain thresholds in women with chronic pelvic pain. Curr Opin Obstet Gynecol. 2014;26(4):253-259.

20. Woolf CJ. Central sensitization: implications for the diagnosis and treatment of pain. Pain. 2011;152(3 suppl):S2-S15.

21. Phillips K, Clauw DJ. Central pain mechanisms in chronic pain states--maybe it is all in their head. Best Pract Res Clin Rheumatol. 2011;25(2):141-154.

22. Latremoliere A, Woolf CJ. Central sensitization: a generator of pain hypersensitivity by central neural plasticity. J Pain. 2009;10(9):895-926.

23. Brawn J, Morotti M, Zondervan KT, Becker CM, Vincent K. Central changes associated with chronic pelvic pain and endometriosis. Hum Reprod Update. 2014;20(5):737-747.

24. Lai HH, Gardner V, Ness TJ, Gereau RW. Segmental hyperalgesia to mechanical stimulus in interstitial cystitis/bladder pain syndrome: evidence of central sensitization. J Urol. 2014;191(5):1294-1299.

25. Verne GN, Price DD. Irritable bowel syndrome as a common precipitant of central sensitization. Curr Rheumatol Rep. 2002;4(4):322-328.

26. Kaya S, Hermans L, Willems T, Roussel N, Meeus M. Central sensitization in urogynecological chronic pelvic pain: a systematic literature review. Pain Physician. 2013;16(4):291-308.

27. Iacovides S, Avidon I, Baker FC. What we know about primary dysmenorrhea today: a critical review. Hum Reprod Update. 2015;21(6): $762-778$
28. Marjoribanks J, Ayeleke RO, Farquhar C, Proctor M. Nonsteroidal anti-inflammatory drugs for dysmenorrhoea. Cochrane Database Syst Rev. 2015;7:CD001751.

29. Wong CL, Farquhar C, Roberts H, Proctor M. Oral contraceptive pill for primary dysmenorrhoea. Cochrane Database Syst Rev. 2009;(4):CD002120.

30. Wong CL, Farquhar C, Roberts H, Proctor M. Oral contraceptive pill as treatment for primary dysmenorrhoea. Cochrane Database Syst Rev. 2009;(2):CD002120.

31. Yu A. Complementary and alternative treatments for primary dysmenorrhea in adolescents. Nurse Pract. 2014;39(11):1-12.

32. Molins-Cubero S, Rodriguez-Blanco C, Oliva-Pascual-Vaca A, HerediaRizo AM, Bosca-Gandia JJ, Ricard F. Changes in pain perception after pelvis manipulation in women with primary dysmenorrhea: a randomized controlled trial. Pain Med. 2014;15(9):1455-1463.

33. Payne LA, Rapkin AJ, Lung KC, Seidman LC, Zeltzer LK, Tsao JC. Pain catastrophizing predicts menstrual pain ratings in adolescent girls with chronic pain. Pain Med. 2016;17(1):16-24.

34. Cox DJ, Meyer RG. Behavioral treatment parameters with primary dysmenorrhea. J Behav Med. 1978;1(3):297-310.

35. Giamberardino MA, Berkley KJ, Iezzi S, de Bigontina P, Vecchiet L. Pain threshold variations in somatic wall tissues as a function of menstrual cycle, segmental site and tissue depth in non-dysmenorrheic women, dysmenorrheic women and men. Pain. 1997;71(2):187-197.

36. Bajaj P, Bajaj P, Madsen H, Arendt-Nielsen L. A comparison of modality-specific somatosensory changes during menstruation in dysmenorrheic and nondysmenorrheic women. Clin J Pain. 2002;18(3):180-190.

37. Iacovides S, Baker FC, Avidon I, Bentley A. Women with dysmenorrhea are hypersensitive to experimental deep muscle pain across the menstrual cycle. J Pain. 2013;14(10):1066-1076.

38. Lee LC, Tu CH, Chen LF, et al. Association of brain-derived neurotrophic factor gene Val66Met polymorphism with primary dysmenorrhea. PLoS One. 2014;9(11):e112766.

39. Wei SY, Chen LF, Lin MW, et al. The OPRM1 A118G polymorphism modulates the descending pain modulatory system for individual pain experience in young women with primary dysmenorrhea. Sci Rep. 2017;7:39906.

40. Arendt-Nielsen L, Madsen H, Jarrell J, Gregersen H, Drewes AM. Pain evoked by distension of the uterine cervix in women with dysmenorrhea: evidence for central sensitization. Acta Obstet Gynecol Scand. 2014;93(8):741-748.

41. Brinkert W, Dimcevski G, Arendt-Nielsen L, Drewes AM, Wilder-Smith $\mathrm{OH}$. Dysmenorrhoea is associated with hypersensitivity in the sigmoid colon and rectum. Pain. 2007;132(suppl 1):S46-S51.

42. Kaislasuo J, Heikinheimo O, Lahteenmaki P, Suhonen S. Predicting painful or difficult intrauterine device insertion in nulligravid women. Obstet Gynecol. 2014;124(2 pt 1):345-353.

43. Slater H, Paananen M, Smith AJ, et al. Heightened cold pain and pressure pain sensitivity in young female adults with moderate-to-severe menstrual pain. Pain. 2015;156(12):2468-2478.

44. Haman JO. Pain threshold in dysmenorrhea. Am J Obstet Gynecol. 1944;47:686-691.

45. Granot M, Yarnitsky D, Itskovitz-Eldor J, Granovsky Y, Peer E, Zimmer EZ. Pain perception in women with dysmenorrhea. Obstet Gynecol. 2001;98(3):407-411.

46. Goolkasian P. An ROC analysis of pain reactions in dysmenorrheic and nondysmenorrheic women. Percept Psychophys. 1983;34(4):381-386.

47. Iacovides S, Avidon I, Baker FC. Women with dysmenorrhoea are hypersensitive to experimentally induced forearm ischaemia during painful menstruation and during the pain-free follicular phase. Eur $J$ Pain. 2015;19(6):797-804.

48. Tu FF, Epstein AE, Pozolo KE, Sexton DL, Melnyk AI, Hellman KM. A noninvasive bladder sensory test supports a role for dysmenorrhea increasing bladder noxious mechanosensitivity. Clin J Pain. 2013;29(10):883-890.

49. Aberger EW, Denney DR, Hutchings DF. Pain sensitivity and coping strategies among dysmenorrheic women: much ado about nothing. Behav Res Ther. 1983;21(2):119-127. 
50. Hapidou EG, De Catanzaro D. Sensitivity to cold pressor pain in dysmenorrheic and non-dysmenorrheic women as a function of menstrual cycle phase. Pain. 1988;34(3):277-283.

51. Amodei N, Nelson-Gray RO. Reactions of dysmenorrheic and nondysmenorrheic women to experimentally induced pain throughout the menstrual cycle. J Behav Med. 1989;12(4):373-385.

52. Rezaii T, Hirschberg AL, Carlstrom K, Ernberg M. The influence of menstrual phases on pain modulation in healthy women. J Pain. 2012;13(7): 646-655.

53. Rhudy JL, Bartley EJ. The effect of the menstrual cycle on affective modulation of pain and nociception in healthy women. Pain. 2010;149(2): 365-372.

54. Rhudy JL, Bartley EJ, Palit S, et al. Do sex hormones influence emotional modulation of pain and nociception in healthy women? Biol Psychol. 2013;94(3):534-544.

55. Riley JL 3rd, Robinson ME, Wise EA, Price DD. A meta-analytic review of pain perception across the menstrual cycle. Pain. 1999;81(3):225-235.

56. Institute of Medicine. Relieving Pain in America: A Blueprint for Transforming Prevention, Care, Education and Research. Washington, DC: National Academies Press; 2011.
57. Iacovides $\mathrm{S}$, Avidon I, Baker FC. Does pain vary across the menstrual cycle? A review. Eur J Pain. 2015;19(10):1389-1405.

58. Rezaii T, Ernberg M. Influence of oral contraceptives on endogenous pain control in healthy women. Exp Brain Res. 2010;203(2):329-338.

59. Veldhuijzen DS, Keaser ML, Traub DS, Zhuo J, Gullapalli RP, Greenspan JD. The role of circulating sex hormones in menstrual cycle-dependent modulation of pain-related brain activation. Pain. 2013;154(4): $548-559$.

60. Vincent K, Warnaby C, Stagg CJ, Moore J, Kennedy S, Tracey I. Brain imaging reveals that engagement of descending inhibitory pain pathways in healthy women in a low endogenous estradiol state varies with testosterone. Pain. 2013;154(4):515-524.

61. Paller CJ, Campbell CM, Edwards RR, Dobs AS. Sex-based differences in pain perception and treatment. Pain Med. 2009;10(2):289-299.

62. Yarnitsky D. Conditioned pain modulation (the diffuse noxious inhibitory control-like effect): its relevance for acute and chronic pain states. Curr Opin Anaesthesiol. 2010;23(5):611-615.

63. Yarnitsky D, Arendt-Nielsen L, Bouhassira D, et al. Recommendations on terminology and practice of psychophysical DNIC testing. Eur $J$ Pain. 2010;14(4):339.
Journal of Pain Research

\section{Publish your work in this journal}

The Journal of Pain Research is an international, peer reviewed, open access, online journal that welcomes laboratory and clinical findings in the fields of pain research and the prevention and management of pain. Original research, reviews, symposium reports, hypothesis formation and commentaries are all considered for publication.

\section{Dovepress}

The manuscript management system is completely online and includes a very quick and fair peer-review system, which is all easy to use. Visit http://www.dovepress.com/testimonials.php to read real quotes from published authors. 